\title{
Searches for Tau Sleptons at the Large Hadron Collider
}

\author{
Alexander Mann* \\ on behalf of the ATLAS and CMS collaborations \\ Ludwig-Maximilians-Universität München \\ E-mail: a.mann@lmu.de
}

\begin{abstract}
Supersymmetry is a hypothetical extension of the Standard Model of particle physics which helps answer a number of open questions arising from our current understanding of the workings of the universe at the fundamental level. In its minimal implementation, this additional postulated symmetry between fermions and bosons predicts the existence of a host of new particles which are the focus of an extensive search program at the Large Hadron Collider.

In these proceedings, an overview is presented of the progress made in searches which look for either direct production of scalar tau leptons or production of supersymmetric particles which are assumed to decay through scalar tau leptons. The decays of scalar tau leptons to their Standard Model partners lead to challenging experimental signatures, requiring dedicated reconstruction algorithms which are usually based on machine-learning techniques. The latest results from the ATLAS and CMS collaborations are presented, making use of the large dataset of proton-proton collisions at a center-of-mass energy of $\sqrt{s}=13 \mathrm{TeV}$ provided by the Large Hadron Collider.
\end{abstract}

7th Annual Conference on Large Hadron Collider Physics — LHCP2019

20th - 25th May, 2019

Puebla, Mexico

${ }^{*}$ Speaker. 


\section{Introduction}

Supersymmetry (SUSY) is a hypothetical extension of the Standard Model of particle physics, that is appealing due to the simplicity of its underlying theoretical idea, its predictiveness and the fact that it allows to answer a number of open questions in the Standard Model. Supersymmetry complements the Standard Model by an additional symmetry that relates fermions and bosons. It thereby proposes a solution to the fine-tuning problem of the Higgs mass, allows for a unification of the gauge coupling constants of the strong and electroweak force at the energy scale of Grand Unified Theories, and it also provides a weakly-interacting massive particle as a candidate for cold dark matter. In the Minimal Supersymmetric Standard Model (MSSM) as the consistent extension of the Standard Model with the smallest number of additional particles, the particle content roughly doubles with respect to the known spectrum of elementary particles. All of these new particles are being searched for at the Large Hadron Collider (LHC, [1]) using proton-proton collision data that is recorded, among others, by the two big experiments, the ATLAS detector [2] and the CMS detector [3].

Part of this extensive search program are searches for tau sleptons $(\widetilde{\tau})$, the supersymmetric scalar partners of the tau leptons of the Standard Model. The chiral supermultiplets for the third generation of charged leptons contain a scalar partner for each of the left- and right-handed chirality components of the tau lepton. These two new particles, denoted $\widetilde{\tau}_{L}$ and $\widetilde{\tau}_{R}$, can mix and give rise to a lighter mass eigenstate $\widetilde{\tau}_{1}$ and a heavier state $\widetilde{\tau}_{2}$. The kinematics of the products from the decay of the tau slepton into a tau lepton and a neutralino, $\widetilde{\tau} \rightarrow \tau \widetilde{\chi}^{0}$, depends on the mixing of the $\widetilde{\tau}$ and nature of the $\widetilde{\chi}^{0}$, which is itself a mixed state of the supersymmetric partners of the Higgs bosons and electroweak gauge bosons of the MSSM. Production of tau sleptons at the LHC can happen directly via a $Z$ boson or photon in quark-antiquark annihilation as shown in the right-most diagram in Figure 1. The other diagrams in Figure 1 show how tau sleptons can be produced indirectly in the decay chains of supersymmetric particles such as gluinos $(\widetilde{g})$, top squarks $\left(\tilde{t}_{1}\right)$, charginos $\left(\widetilde{\chi}_{1}^{ \pm}\right)$ and neutralinos $\left(\widetilde{\chi}_{2}^{0}\right)$.

The cross sections for the production of these supersymmetric particles vary over many orders of magnitude and depend on their couplings and their masses as shown in Figure 2. Supersymmetric particles that carry color charge have the largest cross sections. The figure shows the cross sections for production of gluino pairs, squark pairs $\widetilde{q} \widetilde{q}^{*}$ (summing over the first and the second generation of squarks and bottom squarks, which are here assumed to be mass-degenerate), and top-squark pairs.
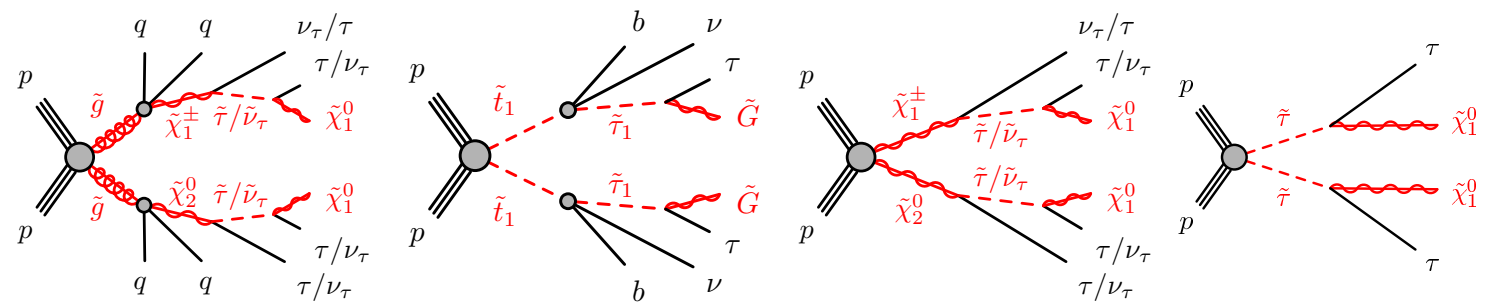

Figure 1: Diagrams of production of pairs of tau sleptons in proton-proton collisions. From left to right: indirect production in decay chains following the production of gluino pairs, top-squark pairs and pairs of electroweak gauginos; direct production. 


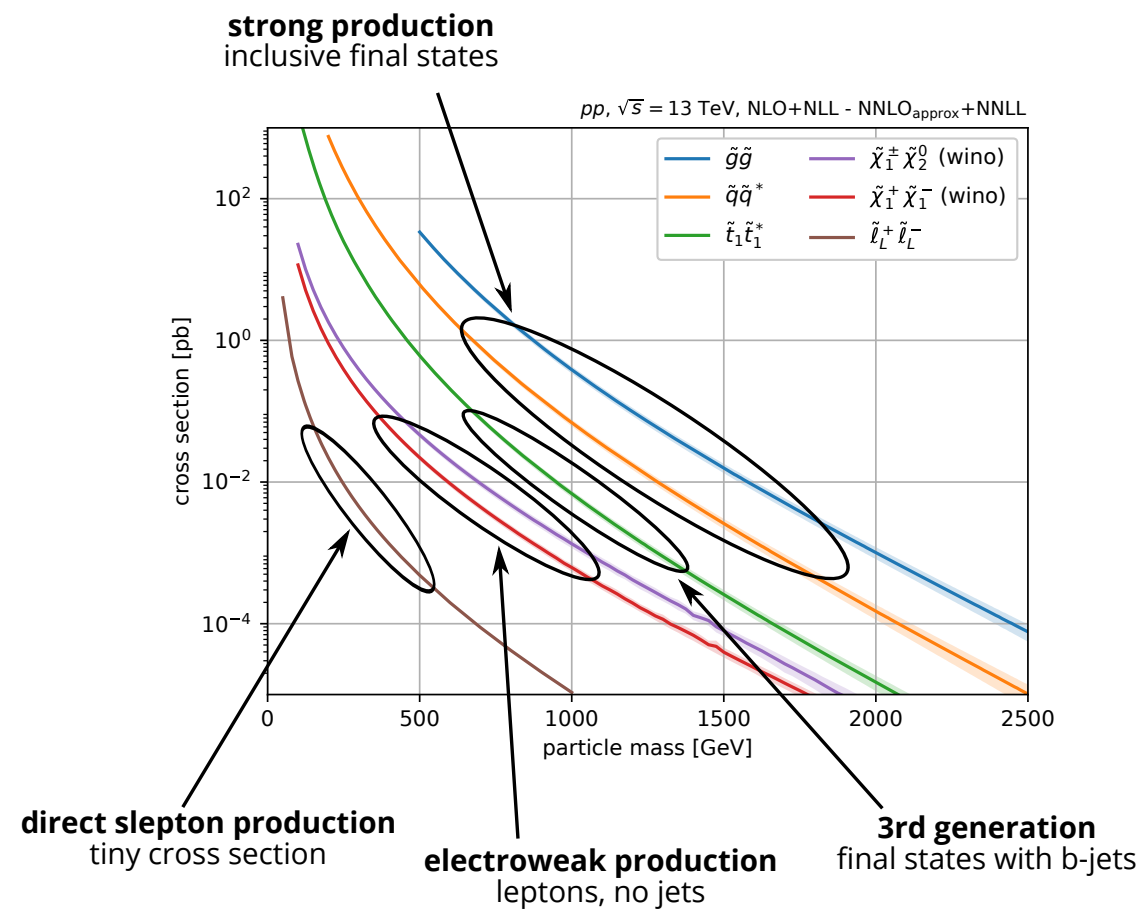

Figure 2: Cross sections for the production of various supersymmetric particles in proton-proton collisions at a center-of-mass energy of $\sqrt{s}=13 \mathrm{TeV}$ as function of the (assumed) mass of the particles [4-12]; values taken from $[13,14]$.

The production cross sections via the electroweak interaction for charginos and neutralinos are considerably smaller, and yet smaller is that of direct production of sleptons $(\widetilde{\ell})$ through a DrellYan process. This also determines the order in which the experiments at the LHC have become sensitive to the various supersymmetric particles and the mass reach of the exclusion limits for these particles in simplified-model interpretations. Simplified models are widely used now to interpret the observations in searches for new particles not only for supersymmetric scenarios. They only consider a specific production mode and decay chain and make additional simplifying assumptions on the branching ratios and masses so that the number of free parameters is reduced a lot. The free parameters are usually chosen to be two of the masses of the new particles so that the sensitivity of a search can be displayed in terms of the exclusion reach on the mass assumptions.

In these proceedings, results from recent searches for direct and indirect production of tau sleptons by both the ATLAS and CMS collaborations are presented. All of these searches assume supersymmetric particles to decay promptly after their production, and $R$-parity to be conserved. The latter means supersymmetric particles are always produced in pairs, supersymmetric particles have an odd number of supersymmetric particles among their decay products, and the lightest supersymmetric particle is stable. Section 2 covers searches for indirect production of tau sleptons, ordered by decreasing production cross sections. As discussed below, most of these searches do not use the mass of the tau slepton directly as a free parameter of the interpretation but the tau slepton appears as an intermediate particle in the decay chain of the supersymmetric particles produced in the proton-proton collisions. Afterwards, in Section 3 the latest searches for direct production of 

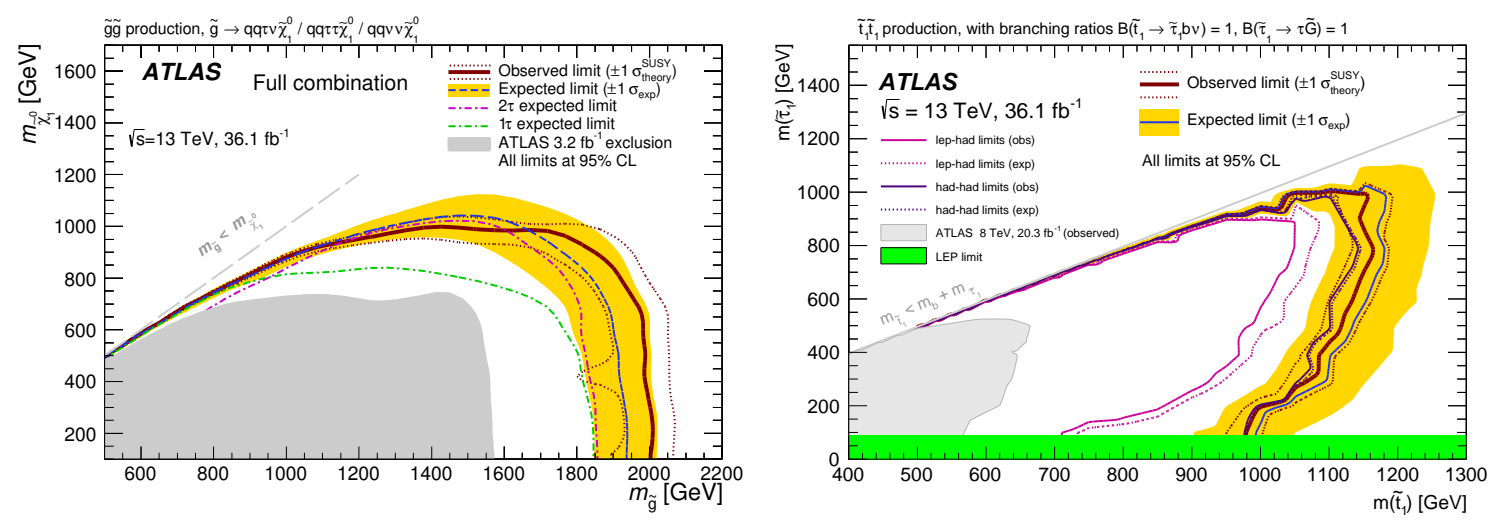

Figure 3: Exclusion contours from the ATLAS search for squarks and gluinos in final states with hadronically decaying tau leptons (left, [15]) and from the ATLAS search for top squarks decaying to tau sleptons (right, [16]). Both searches use $36.1 \mathrm{fb}^{-1}$ of proton-proton collision data taken in 2015 and 2016. In addition to the expected and observed limits from the full analysis, both plots show the exclusion reach of the two respective individual channels.

tau sleptons are highlighted. These are the first analyses that have sensitivity to direct production of tau sleptons at the LHC. Section 4 summarizes two prospective studies by ATLAS and CMS on their sensitivity to direct tau-slepton production at the high-luminosity upgrade of the LHC (HL-LHC), before Section 5 closes with a brief set of conclusions and an outlook.

\section{Searches for Indirect Production of Tau Sleptons}

The ATLAS search for squarks and gluinos in final states with hadronically decaying tau leptons [15] targets final states with either exactly one or at least two hadronically decaying tau leptons, and two or more jets and missing transverse momentum. As the observed data is in agreement with the expected event yields from the Standard Model backgrounds, limits are set at $95 \%$ confidence level (CL) in a simplified model with gluino-pair production shown in the left-most diagram in Figure 1. The limits on the mass of the gluino $\widetilde{g}$ and the mass of the lightest neutralino $\widetilde{\chi}_{1}^{0}$ are shown in the left plot in Figure 3. The mass of the tau slepton is not used as a free parameter in this model, but set to lie half-way between the mass $m\left(\widetilde{\chi}_{1}^{ \pm}, \widetilde{\chi}_{2}^{0}\right)$ of the chargino $\widetilde{\chi}_{1}^{ \pm}$and second-lightest neutralino $\widetilde{\chi}_{2}^{0}$, which are assumed to be mass-degenerate, and the mass of the lightest neutralino $\widetilde{\chi}_{1}^{0}$. In turn, the mass $m\left(\widetilde{\chi}_{1}^{ \pm}, \widetilde{\chi}_{2}^{0}\right)$ is set half-way between the mass of the gluino $\widetilde{g}$ and again the mass of the lightest neutralino $\widetilde{\chi}_{1}^{0}$. The paper also includes an additional interpretation of the search results in a model of gauge-mediated SUSY breaking.

In contrast to the inclusive search for squarks and gluinos in final states with tau leptons, the search for top squarks decaying to tau sleptons by the ATLAS collaboration [16] uses the tauslepton mass as a parameter in the interpretation. In this search, only three supersymmetric particles are assumed to be light enough to be relevant for the phenomenology at the LHC, the top squark $\tilde{t}$, the tau slepton $\widetilde{\tau}$, and the gravitino $\widetilde{G}$ as the nearly massless lightest supersymmetric particle. The top squark decays via a 3-body decay mediated by an off-shell chargino to a $b$ quark, a tau neutrino and the tau slepton as shown in the second diagram in Figure 1, where the tau slepton further decays to the tau lepton and the gravitino. This final state yields an intriguing detector signature 

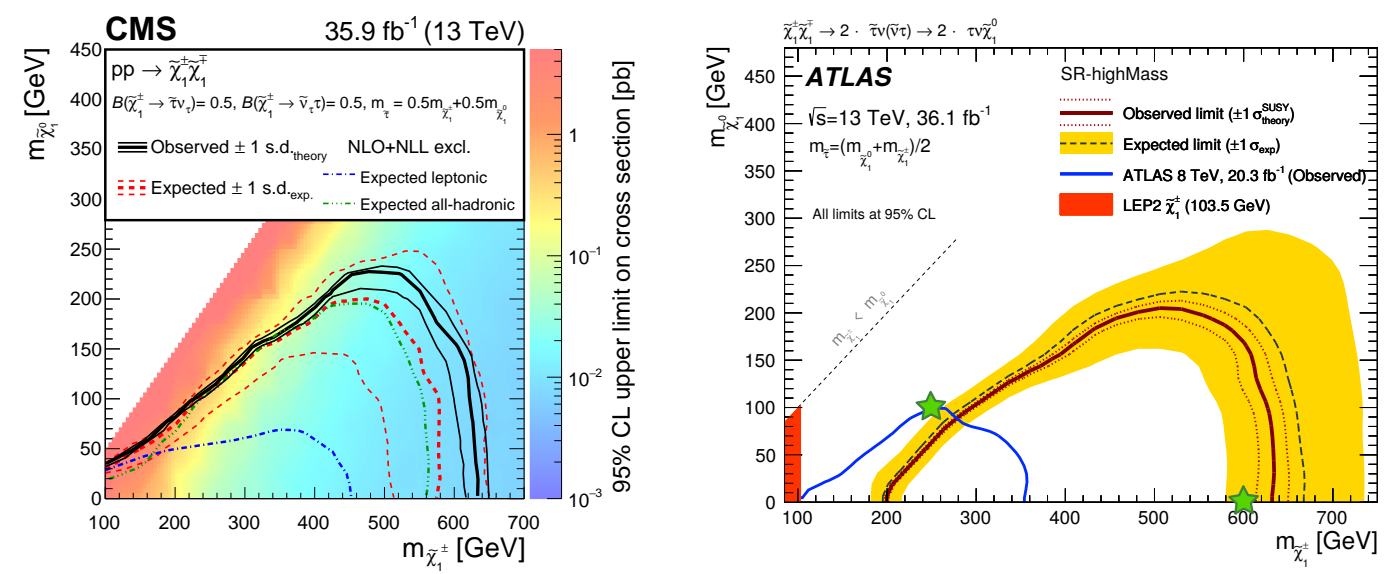

Figure 4: Exclusion contours from the searches for production of chargino pairs with decays to the lightest neutralino via tau sleptons or tau sneutrinos by CMS (left, [21]) and ATLAS (right, [22]). Both searches use $36 \mathrm{fb}^{-1}$ of proton-proton collision data. The green stars in the right-hand plot mark two benchmark scenarios that have been used for additional studies on the impact of the assumption on the mass of the tau slepton discussed in the text.

with two $b$-jets, two tau leptons and large missing transverse momentum. The analysis defines signal selections based mainly on the missing transverse momentum and the stransverse mass [1719] and uses a statistical combination of two decay channels, where either both tau leptons decay hadronically (had-had) or one tau lepton decays hadronically and the other leptonically (lep-had). As no significant excess is observed, the search results yield exclusion limits on the mass of the top squark and tau slepton as shown in the right plot in Figure 3. The limits in this particular simplified model extend to $m\left(\widetilde{\tau}_{1}\right)=1.0 \mathrm{TeV}$ and $m\left(\tilde{t}_{1}\right)=1.16 \mathrm{TeV}$, and are the strongest on both of these particles at the time of writing. CMS has published a similar analysis [20] for LHCP2019 that is discussed elsewhere in these proceedings. The tau-slepton mass is not a direct parameter in the interpretation of the CMS search for top-squark pair production in a di-tau final state, and top-squark masses up to $1.1 \mathrm{TeV}$ are excluded.

Both ATLAS and CMS have published searches for the electroweak production of charginos and neutralinos in final states with tau leptons. These searches are based on a dataset of $36 \mathrm{fb}^{-1}$ taken in 2015 and 2016 (ATLAS) or 2016 (CMS) and consider final states with two tau leptons and missing transverse momentum but no jets with large transverse momentum. The CMS analysis [21] covers all possible decays of the two tau leptons except for same-flavor light-lepton pairs (electrons or muons). Three signal selections target events with exactly two hadronically decaying tau leptons $\left(\tau_{h}\right)$, another 44 are defined for each of the $e \tau_{h}, \mu \tau_{h}$ and $e \mu$ channels. The ATLAS pendant [22] only considers events in which two hadronically decaying tau leptons are reconstructed and employs two signal selections targeting scenarios with small and large mass differences between the chargino $\widetilde{\chi}_{1}^{ \pm}$and neutralino $\widetilde{\chi}_{1}^{0}$. Data-driven approaches are used in both publications to estimate the dominant Standard Model backgrounds arising from multi-jet and $W+$ jets production.

No significant deviations from the expected SM background are observed. The results are interpreted in a simplified model with $\widetilde{\chi}_{1}^{ \pm} \widetilde{\chi}_{1}^{\mp}$ production, similar to the third diagram in Figure 1 but with the $\widetilde{\chi}_{2}^{0}$ branch replaced by a $\widetilde{\chi}_{1}^{\mp}$ and its corresponding decay via $\tilde{\tau} / \widetilde{v}_{\tau}$ as in the upper 

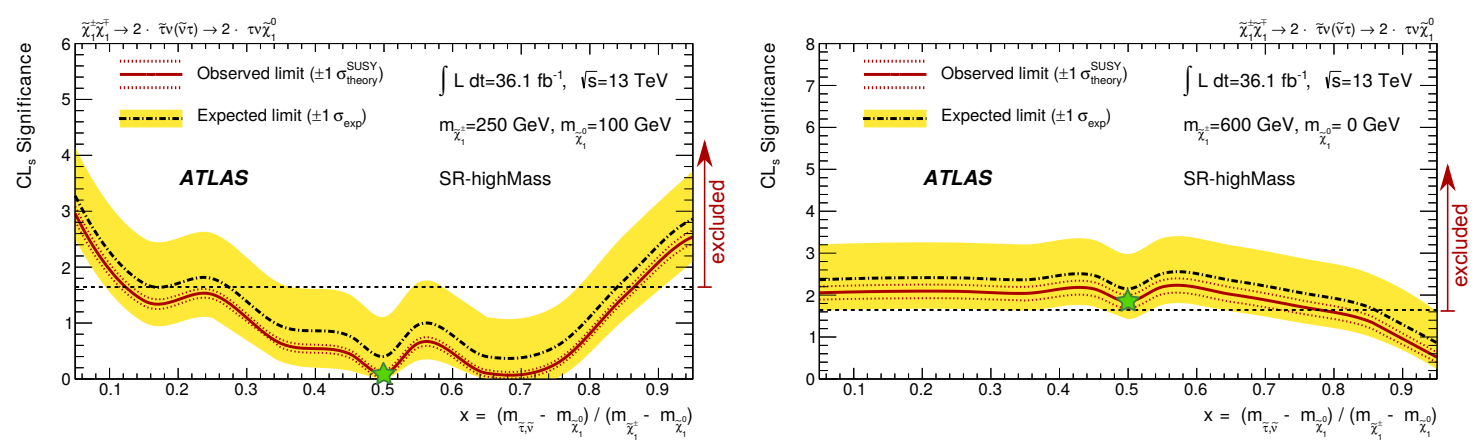

Figure 5: ATLAS study on the impact of the assumption on the mass of the tau slepton (and tau sneutrino) on the exclusion sensitivity for two benchmark points in the simplified model with $\widetilde{\chi}_{1}^{ \pm} \tilde{\chi}_{1}^{\mp}$ production [22]. The green stars mark the location of these benchmark scenarios in the right-hand plot in Figure 4.

branch. The exclusion contours are shown in Figure 4 for CMS and ATLAS, respectively. As can be seen comparing the blue and the green to the red line, the expected exclusion for CMS is driven by the $\tau_{h} \tau_{h}$ channel, but the combination with the leptonic channels yields an improvement of about $20 \mathrm{GeV}$, even though by themselves they have a far weaker reach. The expected exclusion reach extends to higher masses for ATLAS, but due to an upwards fluctuation of the data in the signal selection used by ATLAS for the simplified model with $\widetilde{\chi}_{1}^{ \pm} \widetilde{\chi}_{1}^{\mp}$ production and a deficit in the CMS data with respect to the expected Standard Model backgrounds, ATLAS and CMS report the same observed exclusion limit on the chargino mass of up to $630 \mathrm{GeV}$ for a massless neutralino.

Both ATLAS and CMS give a second interpretation in a simplified model with associated production of the lighter chargino $\widetilde{\chi}_{1}^{ \pm}$and second-lightest neutralino $\widetilde{\chi}_{2}^{0}$, corresponding to the third diagram in Figure 1. However, the published interpretations are not directly comparable: ATLAS adds up signal events from $\widetilde{\chi}_{1}^{ \pm} \widetilde{\chi}_{2}^{0}$ and $\widetilde{\chi}_{1}^{ \pm} \widetilde{\chi}_{1}^{\mp}$ production, the idea being that in a model with a mass-degenerate $\widetilde{\chi}_{1}^{ \pm}$and $\widetilde{\chi}_{2}^{0}$, whenever $\widetilde{\chi}_{1}^{ \pm} \widetilde{\chi}_{2}^{0}$ production is relevant, so will be $\widetilde{\chi}_{1}^{ \pm} \widetilde{\chi}_{1}^{\mp}$ production. In their model, the $\widetilde{\chi}_{2}^{0}$ decays with equal probability via either the tau slepton or the tau sneutrino, so that it contributes either two or zero tau leptons to the final state. This leads to a loss in the signal acceptance of the analysis selection when requiring at least two tau leptons in the final state. CMS, on the other hand, shows the results for $\widetilde{\chi}_{1}^{ \pm} \widetilde{\chi}_{2}^{0}$ production only but they only consider decays of the $\widetilde{\chi}_{2}^{0}$ via tau sleptons, so that they always have three tau leptons in the final state after $\widetilde{\chi}_{1}^{ \pm} \widetilde{\chi}_{2}^{0}$ production.

Both ATLAS and CMS do not choose the mass of the tau slepton (and tau sneutrino) as a free parameter of the simplified model, but set it half-way between the mass parameters $m\left(\widetilde{\chi}_{1}^{ \pm}\right)$and $m\left(\widetilde{\chi}_{1}^{0}\right)$. However, ATLAS has an additional study of the impact of this assumption on the exclusion limit. Defining $x=\left(m\left(\widetilde{\tau}, \widetilde{v}_{\tau}\right)-m\left(\widetilde{\chi}_{1}^{0}\right)\right) /\left(m\left(\widetilde{\chi}_{1}^{ \pm}\right)-m\left(\widetilde{\chi}_{1}^{0}\right)\right)$, the nominal "half-way" assumption in the interpretation corresponds to $x=0.5$. Larger $x$ values bring the mass of the tau slepton closer to the mass of the chargino; lower $x$ values bring it closer to the mass of the neutralino. Two benchmark scenarios, marked by the green stars in the right-hand plot of Figure 4, are used to study the impact of $x$ on the $\mathrm{CL}_{s}$ significance as shown in Figure 5. Scenarios for which the $\mathrm{CL}_{s}$ significance is larger than 1.64 can be excluded at $95 \% \mathrm{CL}$. The first benchmark point sits close to but outside the exclusion contour in Figure 4, and correspondingly the $\mathrm{CL}_{s}$ significance is below 1.64 in the left-hand plot of Figure 5 for $x=0.5$. Interestingly though, scenarios in which the tau- 

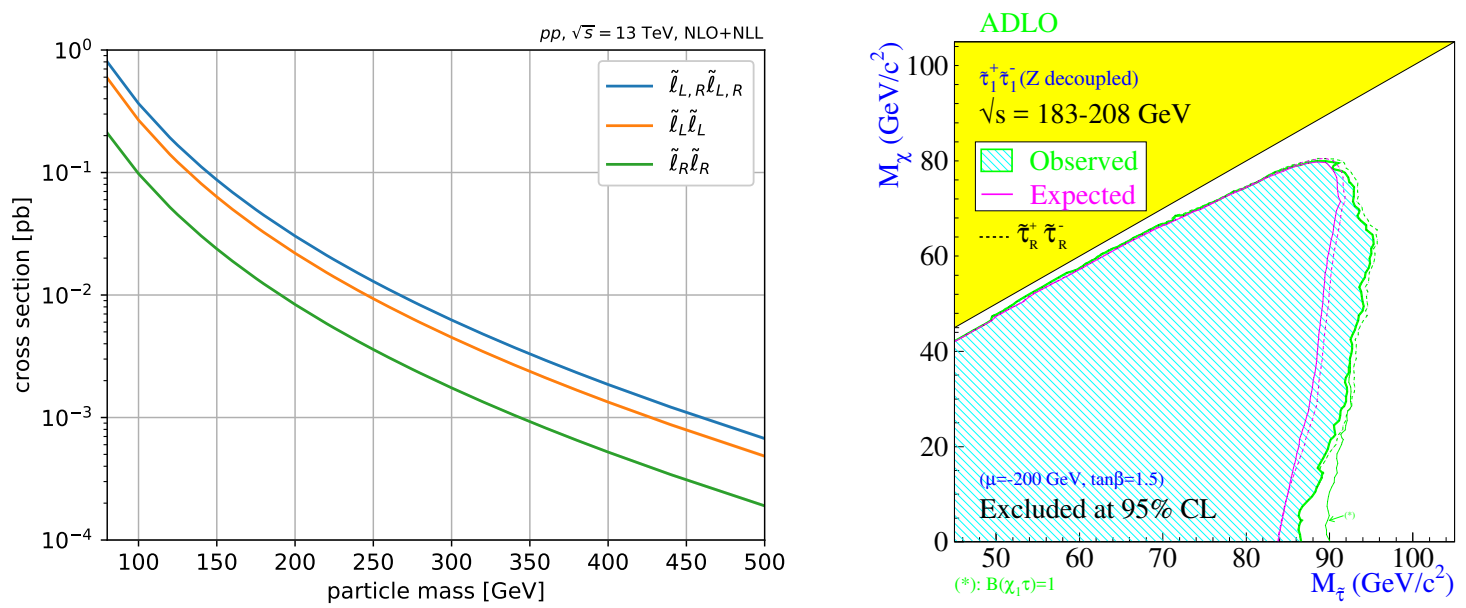

Figure 6: Left: cross sections for direct production of slepton pairs as a function of their mass in protonproton collisions at $\sqrt{s}=13 \mathrm{TeV}[4-8]$; values taken from [13, 14]. Right: expected and observed exclusion domains at $95 \% \mathrm{CL}$ for the production of tau-slepton pairs obtained by the LEP collaborations [23-28].

slepton mass is either very close to the chargino mass (large $x$ ) or neutralino mass (small $x$ ) can be excluded. This is because in this compressed scenario with a small mass splitting $m\left(\widetilde{\chi}_{1}^{ \pm}\right)-m\left(\widetilde{\chi}_{1}^{0}\right)=$ $150 \mathrm{GeV}$, the distribution of the stransverse mass $m_{\mathrm{T} 2}$ of the signal has a well-defined cut-off at $150 \mathrm{GeV}$ if $x=0.5$. For larger or smaller $x$, the $m_{\mathrm{T} 2}$ distribution for the signal events becomes broader, leading to an increased signal acceptance for the same amount of background after the analysis requirement of $m_{\mathrm{T} 2}>90 \mathrm{GeV}$ and thus an increase in the sensitivity and a larger $\mathrm{CL}_{s}$ significance. For the second benchmark point, which sits close to but inside the exclusion contour in the right-hand plot of Figure 4, the mass splitting $m\left(\widetilde{\chi}_{1}^{ \pm}\right)-m\left(\widetilde{\chi}_{1}^{0}\right)$ is large. For most values of $x$ including the nominal, this scenario is excluded at $95 \% \mathrm{CL}$. Only for large $x$ close to 1 , the mass splitting $m\left(\widetilde{\chi}_{1}^{ \pm}\right)-m\left(\widetilde{\tau}, \widetilde{v}_{\tau}\right)$ becomes so small that the reduced transverse momentum of the first tau lepton in the decay chain ${ }^{1}$ from the decay $\widetilde{\chi}_{1}^{ \pm} \rightarrow \widetilde{v}_{\tau} \tau$ leads to a significant loss in the sensitivity of the analysis. The opposite case of small $x \ll 1$ does not have the same effect, as the first tau lepton in the decay chain then naturally has a large transverse momentum due to the small mass of the tau sneutrino in $\widetilde{\chi}_{1}^{ \pm} \rightarrow \widetilde{v}_{\tau} \tau$. But also the light $\widetilde{\tau}$ receives a boost when being produced in the decay of the $\widetilde{\chi}_{1}^{ \pm}$, which is transferred to the second tau lepton from the decay $\widetilde{\tau} \rightarrow \widetilde{\chi}_{1}^{0} \tau$, so that the tau lepton always has a relatively large transverse momentum in this scenario.

\section{Searches for Direct Production of Tau Sleptons}

The direct production of slepton pairs has a particularly low cross section. Its dependence on the mass of the slepton is shown in the left plot in Figure 6. Three curves are shown, for partners of left-handed $\left(\widetilde{\ell}_{L}\right)$ and right-handed leptons $\left(\widetilde{\ell}_{R}\right)$ and the sum for the combined production

\footnotetext{
1 "First tau lepton" and "second tau lepton" here do not refer to the first and second-leading tau lepton, i. e. the tau lepton with the largest or second-largest transverse momentum, but to their position in the decay chain in the simplified model (cf. upper branch in the third diagram in Figure 1): The decay of the chargino produces the first tau lepton if it decays via a tau sneutrino, and the decay of the tau slepton produces the second tau lepton if the chargino decays via a tau slepton. The decay of the chargino can thus only produce either a first or a second tau lepton.
} 
of mass-degenerate sleptons. The production cross section for $\widetilde{\ell}_{L} \widetilde{\ell}_{L}$ is about 2.5 to 3 times larger than that for $\widetilde{\ell}_{R} \widetilde{\ell}_{R}$ if there is no mixing. Limits on the masses of sleptons have been obtained already with data from Run 1 of the LHC [29], where simplified models with direct production of mass-degenerate supersymmetric partners of the electrons and muons (selectrons and smuons) are considered and common values for the left- and right-handed selectron and smuon masses up to $325 \mathrm{GeV}$ are excluded for a massless neutralino. For tau sleptons no sensitivity had been achieved until very recently for several reasons. Assuming selectrons and smuons to have the same mass allows to sum their production cross sections and thus increases the expected signal yields by a factor two compared to production of tau-slepton pairs, which are not usually taken to be massdegenerate with the other two slepton generations. Furthermore, the reconstruction of final states with tau leptons is more difficult than that of final states with electrons or muons. Hadronic and leptonic decays of tau leptons need to be treated separately. The presence of neutrinos from the tau-lepton decays reduces the transverse momentum of the visible decay products and weakens the discriminative power of kinematic variables. And finally, larger probabilities to misidentify other objects like jets as hadronically decaying tau leptons lead to larger backgrounds from multi-jet production and $W+$ jets events.

In this section, two recent results from searches for direct production of tau sleptons at the LHC will be presented. These searches by the ATLAS and CMS collaborations yield the first mass limits in simplified models on either combined pair production of mass-degenerate $\widetilde{\tau}_{L} \widetilde{\tau}_{L}$ and $\widetilde{\tau}_{R} \widetilde{\tau}_{R}$ or production of $\widetilde{\tau}_{L} \widetilde{\tau}_{L}$ only, at least in the sense that they cover a range of mass hypotheses. Searches for this process have been carried out with the dataset from the first run of the LHC taken at $\sqrt{s}=8 \mathrm{TeV}$, and a single model point with a tau-slepton mass of $109 \mathrm{GeV}$ in a scenario with a massless neutralino has been excluded [30] by an improved version of the search for the direct production of charginos, neutralinos and tau sleptons in final states with at least two hadronically decaying tau leptons [31] using multivariate analysis techniques. Before coming to the new results from the LHC, the right plot in Figure 6 shows the expected and observed limits for the production of tau-slepton pairs as function of the tau-slepton and neutralino masses obtained by the LEP collaborations [23-28]. Assuming a branching ratio of $100 \%$ for decays of the tau slepton into a tau lepton and the lightest neutralino, masses of the tau slepton up to $90 \mathrm{GeV}$ are excluded for a massless neutralino in a conservative setting where the partner of the left-handed tau lepton is assumed to be beyond the reach of the experiments. Another search that is relevant in the context of searches for direct production of tau sleptons is the ATLAS search for heavy charged long-lived particles [32]. At variance with all other analyses presented here, in this analysis the tau slepton is assumed to be long-lived enough to leave the detector before it decays. Measurements of the time of flight and the momentum of the tau slepton, which travels significantly slower than the speed of light due to its large mass, allow to conduct an almost background-free search and exclude masses $m\left(\widetilde{\tau}_{R}\right)$ up to $430 \mathrm{GeV}$.

The latest CMS result on direct production of tau sleptons uses the dataset from 2016 and 2017 with a total luminosity of $77.2 \mathrm{fb}^{-1}$ at $\sqrt{s}=13 \mathrm{TeV}$ [33]. It is based on the CMS search for supersymmetry in events with a tau-lepton pair and missing transverse momentum [21] and uses the same techniques to estimate the Standard Model backgrounds. As in this previous analysis, the $\tau_{h} \tau_{h}$ channel is combined with the $\ell \tau_{h}$ channels $(\ell=e$ or $\mu$ ), where now boosted decision trees (BDT) are used for the event selection; the all-leptonic $e \mu$ channel is not used here. In the $\tau_{h} \tau_{h}$ channel, 

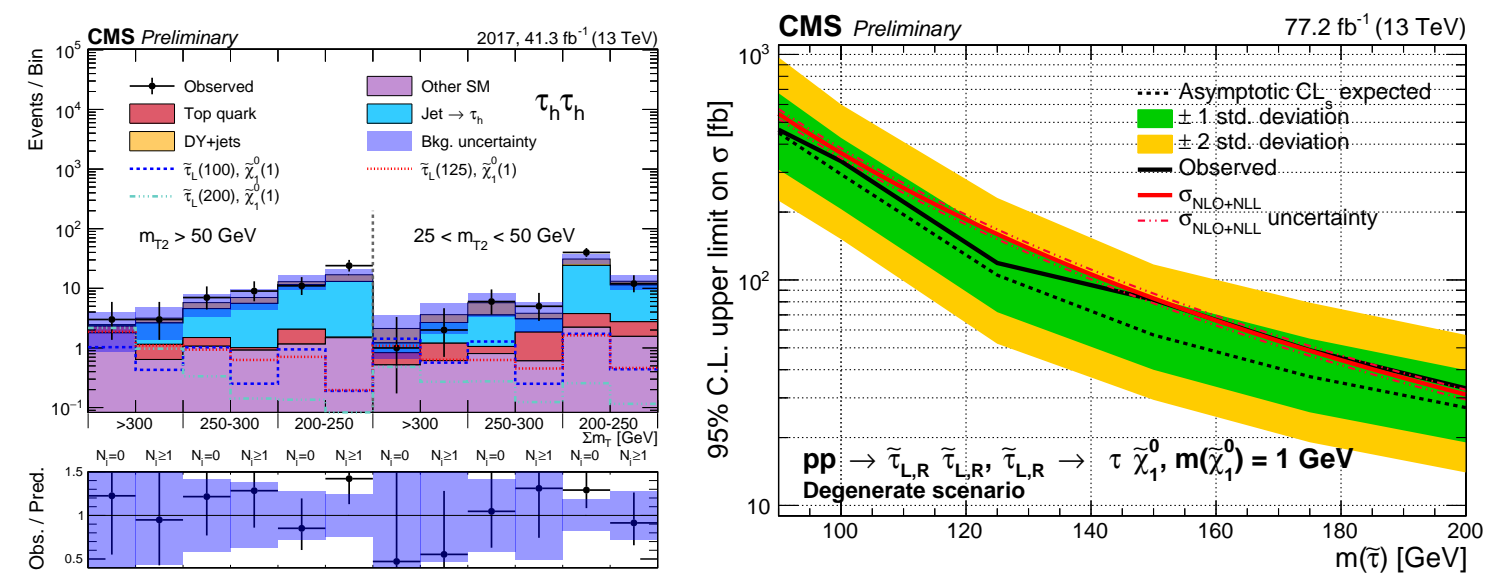

Figure 7: Results from the CMS search for direct tau-slepton pair production in proton-proton collisions at $\sqrt{s}=13 \mathrm{TeV}$ [33]. Left: observed event counts and post-fit predicted yields for the Standard Model backgrounds in the $\tau_{h} \tau_{h}$ channel for the 2017 dataset. The dashed lines show predicted signal yields for three benchmark signal models, where the values in brackets in the legend give the mass of the particle in $\mathrm{GeV}$. Right: excluded cross section for tau-slepton pair production at $95 \% \mathrm{CL}$ in the mass-degenerate scenario for $m\left(\widetilde{\chi}_{1}^{0}\right)=1 \mathrm{GeV}$ using data from 2016 and 2017. The red lines indicate the theoretical signal production cross section and its uncertainty.

events are selected with a di-tau trigger that allows to select tau leptons with an offline transverse momentum down to 40 and $45 \mathrm{GeV}$ in 2016 and 2017 data-taking, respectively. By comparison with the thresholds on the offline transverse momentum that would be required to operate in the efficiency plateau of the ATLAS di-tau trigger, these thresholds are considerably lower. Part of the selection inefficiency of the di-tau trigger is recovered by combining it with a trigger on missing transverse momentum. Six signal selections are defined based on the stransverse mass $m_{\mathrm{T} 2}$ and the sum of the transverse masses of the two tau leptons $\sum m_{\mathrm{T}}$, rejecting events with additional leptons or $b$-jets. Each signal selection groups events with and without jets to form two categories. The resulting observed event counts and predicted yields for the Standard Model backgrounds in the 12 signal selections are shown in the left plot of Figure 7. In the $\ell \tau_{h}$ channels, events are selected with single-lepton triggers, and BDT discriminants are trained on high-level variables for three signal benchmark models with different assumptions on the tau-slepton mass in each of the two channels.

As the data is well described by the predictions for the Standard Model background, limits are set on the cross sections for the direct production of tau sleptons in several scenarios. A full statistical combination of the selected events yields in the $\tau_{h} \tau_{h}$ channel and the full output-score distribution of the BDTs in the $\ell \tau_{h}$ channels is used. The resulting limits are shown in the right plot in Figure 7 for a scenario where it is assumed that the neutralino is almost massless and massdegenerate $\widetilde{\tau}_{L} \widetilde{\tau}_{L}$ and $\widetilde{\tau}_{R} \widetilde{\tau}_{R}$ are produced. It can be seen that in this scenario tau-slepton masses of up to $150 \mathrm{GeV}$ can be excluded at $95 \% \mathrm{CL}$. This is the first time at the LHC that a range of masses is excluded for directly produced tau sleptons. Additional interpretations are given for a scenario with $\widetilde{\tau}_{L} \widetilde{\tau}_{L}$ production only and for masses of the neutralino of 10 and $20 \mathrm{GeV}$, but no exclusion is reported for any of these.

The ATLAS search for direct tau-slepton production in events with two hadronic tau lep- 

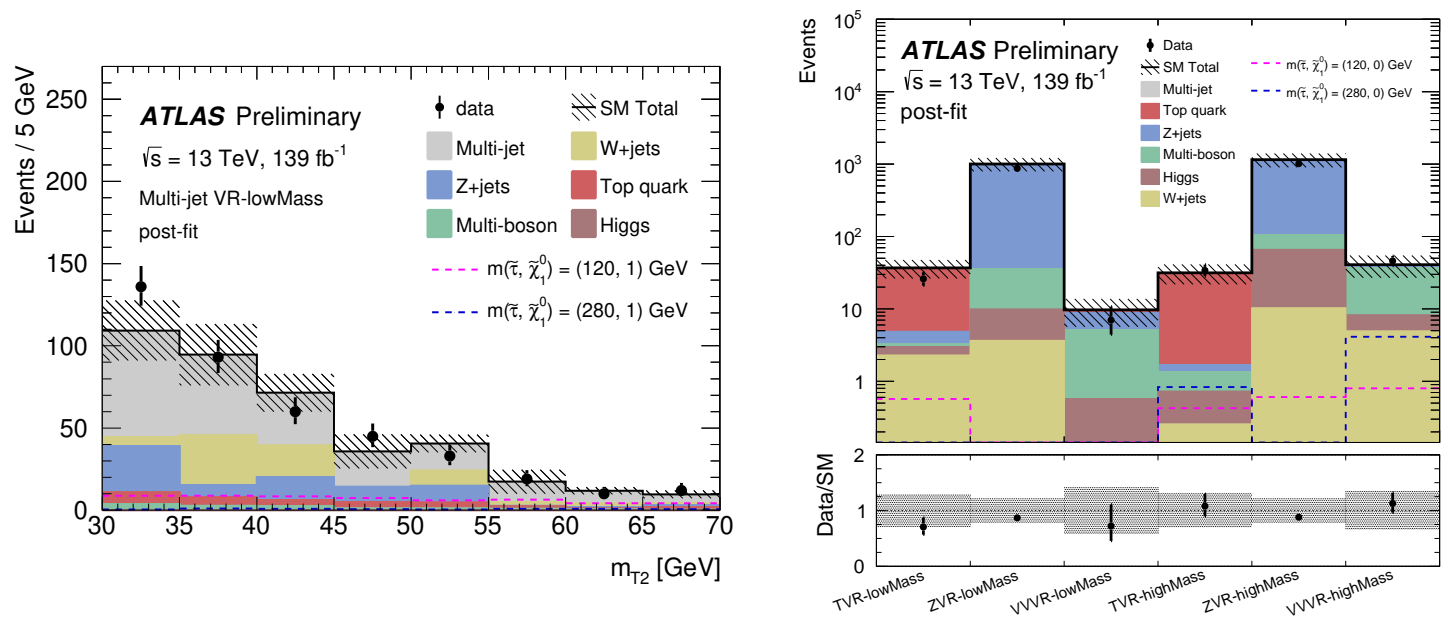

Figure 8: Validation regions from the ATLAS search for direct tau-slepton production in events with two hadronic tau leptons in $\sqrt{s}=13 \mathrm{TeV} p p$ collisions [34]. Left: distribution of $m_{\mathrm{T} 2}$ in the multi-jet background validation region accompanying SR-lowMass. The multi-jet shape is derived using the ABCD method and normalized by a correction factor determined in a fit to data. Right: observed event counts and post-fit predicted yields in the validation regions targeting the background from top-quark (TVR-X), $Z$ boson (ZVR$\mathrm{X}$ ) and diboson (VVVR-X) production. The Standard Model backgrounds other than multi-jet production, which is negligible, are estimated from simulation.

tons [34] is the first search for supersymmetry in final states with hadronically decaying tau leptons to make use of the full ATLAS dataset taken in Run 2 of the LHC with a luminosity of $139 \mathrm{fb}^{-1}$. In this search, only the $\tau_{h} \tau_{h}$ channel is considered. Two non-overlapping signal selections (SRlowMass and SR-highMass) are defined. In SR-lowMass, which targets tau sleptons of low masses, events are selected by an asymmetric di-tau trigger and required to have a modest amount of missing transverse energy $75 \mathrm{GeV}<E_{\mathrm{T}}^{\text {miss }}<150 \mathrm{GeV}$. In SR-highMass, events are selected by a combined trigger on two tau leptons and missing transverse energy. This signal selection targets higher tau-slepton masses and requires offline missing transverse energy $E_{\mathrm{T}}^{\text {miss }}>150 \mathrm{GeV}$. Both signal selections require $m_{\mathrm{T} 2}>70 \mathrm{GeV}$ and the invariant mass of the two tau leptons $m\left(\tau_{1}, \tau_{2}\right)>120 \mathrm{GeV}$ to reduce background from events with $Z$-boson or Higgs-boson production, and reject events with a third lepton or $b$-jet.

The Standard Model background is estimated in a similar way as in the ATLAS search for direct production of charginos and neutralinos in final states with tau leptons [22] but with two new developments. A data-driven fake-factor method has been developed that allows for an independent cross-check of the estimate of the multi-jet background obtained from the ABCD method. The fake factor, i. e. the misidentification probability of a jet as a hadronically decaying tau lepton, is measured in data as a function of the transverse momentum, pseudorapidity and number of charged tracks of the reconstructed tau lepton. It can then be used to predict the number of multi-jet events entering a selection with hadronically decaying tau leptons by extrapolating from suitably chosen sideband regions. The tau-promotion method, another simulation-based fake-factor method, is used to reduce the statistical uncertainty in the estimate of the $W(\tau v)+$ jets background. It recovers 

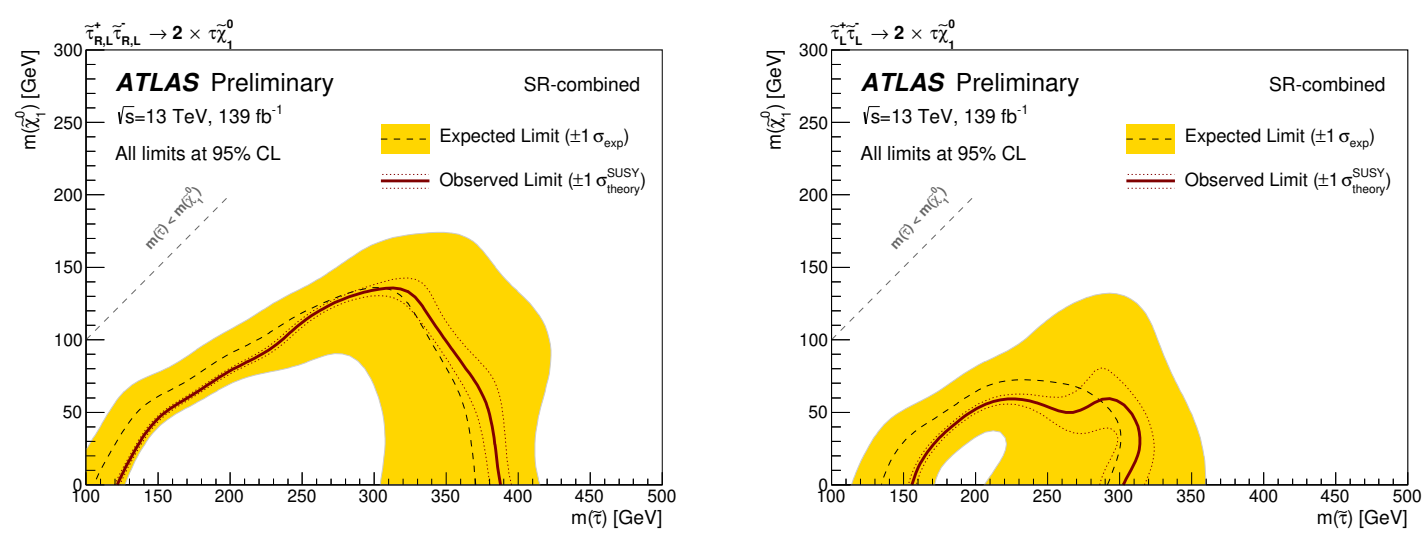

Figure 9: Expected and observed exclusion contours at $95 \% \mathrm{CL}$ in simplified models with production of mass-degenerate $\widetilde{\tau}_{L} \widetilde{\tau}_{L}$ and $\widetilde{\tau}_{R} \widetilde{\tau}_{R}$ (left) and production of $\widetilde{\tau}_{L} \widetilde{\tau}_{L}$ only (right) obtained by the ATLAS collaboration in an analysis of the full Run-2 dataset [34].

simulated events that do not pass the selection requirement of having two tau leptons by picking one jet in every simulated event at random, artificially promoting it to a (fake) tau lepton, and then restoring the correct prediction of the $W(\tau v)+$ jets background yield by reweighting the event sample to the yield of the nominal sample based on a measurement of the fake rates in simulation.

For each of the two signal selections, several additional selections (validation regions) are defined that target different background contributions. The observed event counts in the validation regions are in good agreement with the predicted yields as can be seen in Figure 8. No significant deviation of the data from the expected Standard Model background is also found when applying the signal selections, thus again limits on the presence of new physics are derived from the search results. As the sensitivity of the analysis extends to neutralino masses well beyond the hypothesis of a massless neutralino, the interpretation is not presented in terms of one-dimensional crosssection limits, but the observed and expected number of events in the signal selections are used to place exclusion limits at $95 \% \mathrm{CL}$ on the masses of the tau slepton and lightest neutralino as shown in Figure 9. The left plot in this figure shows the expected and observed limits in a simplified model with production of mass-degenerate $\widetilde{\tau}_{L} \widetilde{\tau}_{L}$ and $\widetilde{\tau}_{R} \widetilde{\tau}_{R}$, where masses between 120 and $390 \mathrm{GeV}$ for a massless lightest neutralino are excluded, and the exclusion contour reaches up to neutralino masses of $135 \mathrm{GeV}$. These limits improve significantly upon the previous result by CMS, in particular for large neutralino and tau-slepton masses. The right plot in the figure shows the expected and observed limits in a simplified model where production of only $\widetilde{\tau}_{L} \widetilde{\tau}_{L}$ is assumed. Here, masses of the tau slepton $\widetilde{\tau}_{L}$ between 160 and $300 \mathrm{GeV}$ are excluded at $95 \% \mathrm{CL}$ for a massless neutralino. This is the first exclusion of direct production of non-degenerate tau sleptons at the LHC. The analysis is also expected to be sensitive to production of $\widetilde{\tau}_{R} \widetilde{\tau}_{R}$ only, but due to a slight excess in the more sensitive signal selection SR-lowMass, this expectation is not realized in the observed limit.

\section{Future Searches for Direct Production of Tau Sleptons}

Both the ATLAS and the CMS collaboration have done prospective studies to evaluate the sensitivity to direct production of tau sleptons that can be reached with the dataset to be taken at 

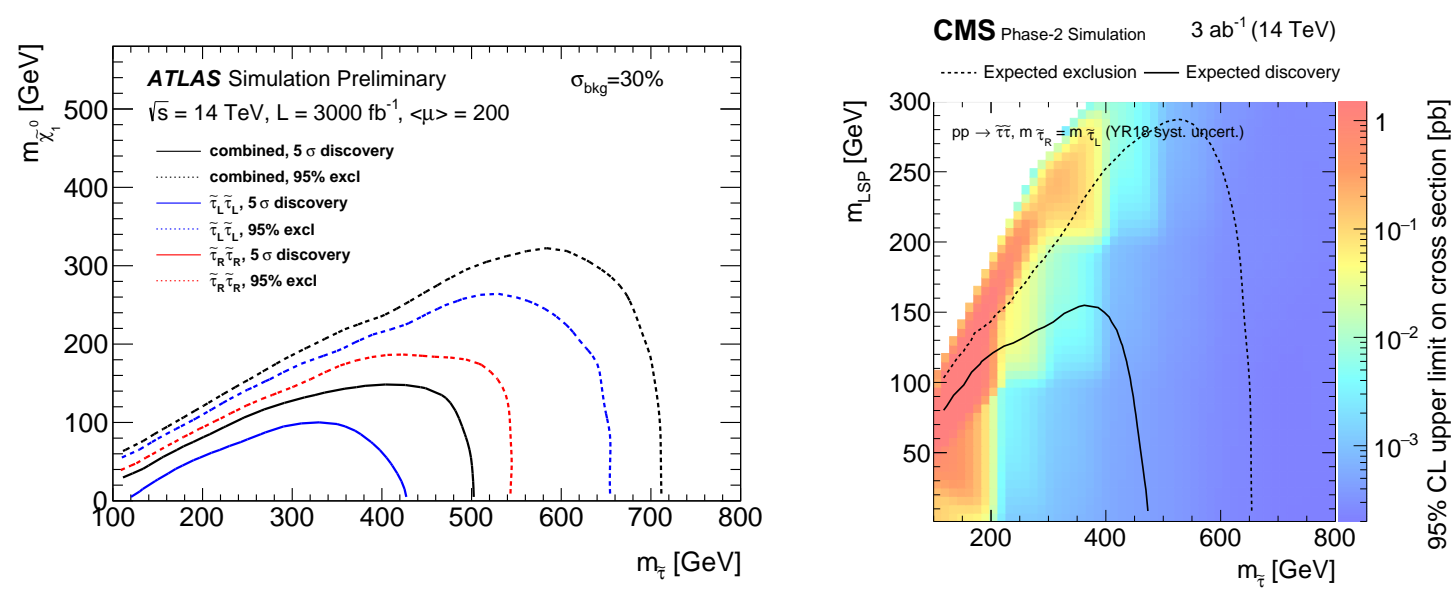

Figure 10: Expected exclusion and discovery sensitivities to direct production of tau-slepton pairs at the HL-LHC for ATLAS (left, [35]) and CMS (right, [36]) as function of the tau-slepton mass and lightest neutralino mass. For the ATLAS plot a systematic uncertainty of $30 \%$ is assumed. The CMS plot includes a systematic uncertainty of $15 \%$ based on a more detailed assessment.

the high-luminosity upgrade of the LHC [37, 38]. These studies assume a dataset of $3 \mathrm{ab}^{-1}$ at a center-of-mass energy $\sqrt{s}=14 \mathrm{TeV}$ and an average number of concurrent proton-proton interactions $\langle\mu\rangle=200$ and use parameterized simulations of the performance of the upgraded ATLAS and CMS detectors. They further assume that the upgraded trigger systems will allow trigger thresholds similar to the triggers currently in use despite the higher $\langle\mu\rangle$. The ATLAS study considers only the $\tau_{h} \tau_{h}$ channel, whereas CMS considers the $\ell \tau_{h}$ and $\tau_{h} \tau_{h}$ channels. The signal selections are based on the stransverse mass $m_{\mathrm{T} 2}$, the sum of the transverse masses of the two tau leptons $\sum m_{\mathrm{T}}$, the transverse momentum of the tau leptons, and the missing transverse energy. Events with additional leptons and in most cases also with jets with large transverse momentum are rejected. The dominant processes contributing to the Standard Model background are production of top-quark pairs and $W+$ jets. Figure 10 shows the expected exclusion and discovery sensitivities for ATLAS and CMS as function of the tau-slepton mass and lightest neutralino mass for decays $\widetilde{\tau} \rightarrow \tau \widetilde{\chi}_{1}^{0}$ with $100 \%$ branching fraction. Despite the different appearance of the plots, the predicted sensitivities are rather similar: for combined pair production of mass-degenerate $\widetilde{\tau}_{L} \widetilde{\tau}_{L}$ and $\widetilde{\tau}_{R} \widetilde{\tau}_{R}$, the expected exclusion sensitivity goes up to $m\left(\widetilde{\tau}_{L, R}\right)=710 \mathrm{GeV}$ for ATLAS and up to $m\left(\widetilde{\tau}_{L, R}\right)=650 \mathrm{GeV}$ for CMS. The experiments would be able to observe tau sleptons with masses up to $m\left(\widetilde{\tau}_{L, R}\right)=500 \mathrm{GeV}$ (ATLAS) or $m\left(\widetilde{\tau}_{L, R}\right)=470 \mathrm{GeV}$ (CMS) with $5 \sigma$ significance. ATLAS also shows the expected exclusion and discovery sensitivities for non-degenerate tau sleptons, reaching up to $m\left(\widetilde{\tau}_{L}\right)=650 \mathrm{GeV}$ and $m\left(\widetilde{\tau}_{R}\right)=540 \mathrm{GeV}$ for the exclusion. The discovery reach for $\widetilde{\tau}_{L}$ goes up to $m\left(\widetilde{\tau}_{L}\right)=430 \mathrm{GeV}$, but a discovery at $5 \sigma$ significance would not be reached for any mass if only $\widetilde{\tau}_{R}$ is produced.

\section{Conclusions and Outlook}

The searches for direct and indirect production of the supersymmetric partners of the tau leptons by the ATLAS and CMS collaborations cover a variety of production modes and a wide range 
of cross sections. None of these searches have reported any significant excess in the events yields above the expected background from Standard Model processes, and the search results are thus interpreted in terms of exclusion limits on production cross sections or as excluded domains in two-dimensional planes spanned by the mass hypotheses for supersymmetric particles that appear in the decay chains of simplified models.

The latest additions to the set of searches are the searches for direct production of tau sleptons, which are extremely difficult due to the small production cross sections and the challenging reconstruction of final states with tau leptons. Two recent searches by ATLAS and CMS show exclusion sensitivity for direct production of tau sleptons over a wide range of masses for the first time at the LHC. There are still obvious opportunities for improvements in the searches for tau sleptons. ATLAS does not yet cover the $\ell \tau_{h}$ channels and CMS still needs to include the dataset from 2018. These improvements likely bring only modest gains, but CMS should then be able to also exclude not only production of mass-degenerate $\widetilde{\tau}_{L} \widetilde{\tau}_{L}$ and $\widetilde{\tau}_{R} \widetilde{\tau}_{R}$ pairs but $\widetilde{\tau}_{L} \widetilde{\tau}_{L}$ production, too. If they are luckier than ATLAS, they may even be able to claim the first sensitivity to $\widetilde{\tau}_{R} \widetilde{\tau}_{R}$ production at the LHC. Additional effort will be needed to close the gaps in the current landscape of exclusion contours, both for compressed scenarios with small mass splittings of the tau slepton and the neutralino into which it decays and for scenarios with light tau-slepton masses. Here, multivariate analysis techniques and shape fits but also improved tau-reconstruction and identification techniques will help. Combinations of analyses from ATLAS and CMS are not common in searches for new physics, but may be worthwhile to try for $\widetilde{\tau}_{R} \widetilde{\tau}_{R}$ production if neither of these experiments can reach sensitivity by itself with the Run-2 dataset. Finally, the trigger thresholds on the transverse momentum of tau leptons are often the bottleneck in the analysis acceptance and thus it is important to work on the triggers for the next period of data-taking at the LHC.

\section{References}

[1] L. Evans and P. Bryant, LHC Machine, JINST 3 (2008) S08001.

[2] ATLAS Collaboration, The ATLAS Experiment at the CERN Large Hadron Collider, JINST 3 (2008) S08003.

[3] CMS Collaboration, The CMS experiment at the CERN LHC, JINST 3 (2008) S08004.

[4] W. Beenakker, C. Borschensky, M. Krämer, A. Kulesza, and E. Laenen, NNLL-fast: predictions for coloured supersymmetric particle production at the LHC with threshold and Coulomb resummation, JHEP 12 (2016) 133, arXiv:1607.07741 [hep-ph] .

[5] G. Bozzi, B. Fuks, and M. Klasen, Transverse-momentum resummation for slepton-pair production at the CERN Large Hadron Collider, Phys. Rev. D 74 (2006) 015001.

[6] G. Bozzi, B. Fuks, and M. Klasen, Threshold resummation for slepton-pair production at hadron colliders, Nucl. Phys. B 777 (2007) 157 - 181.

[7] G. Bozzi, B. Fuks, and M. Klasen, Joint resummation for slepton pair production at hadron colliders, Nucl. Phys. B 794 (2008) 46-60, arXiv:0709.3057 [hep-ph] .

[8] B. Fuks, M. Klasen, D. R. Lamprea, and M. Rothering, Precision predictions for electroweak superpartner production at hadron colliders with Resummino, Eur. Phys. J. C 73 (2013) 2480, arXiv:1304.0790 [hep-ph]. 
[9] J. Debove, B. Fuks, and M. Klasen, Transverse-momentum resummation for gaugino-pair production at hadron colliders, Phys. Lett. B 688 (2010) 208-211, arXiv:0907.1105 [hep-ph] .

[10] J. Debove, B. Fuks, and M. Klasen, Threshold resummation for gaugino pair production at hadron colliders, Nucl. Phys. B 842 (2011) 51 - 85.

[11] J. Debove, B. Fuks, and M. Klasen, Joint resummation for gaugino pair production at hadron colliders, Nucl. Phys. B 849 (2011) 64 - 79.

[12] B. Fuks, M. Klasen, D. R. Lamprea, and M. Rothering, Gaugino production in proton-proton collisions at a center-of-mass energy of 8 TeV, JHEP 10 (2012) 081, arXiv: 1207.2159 [hep-ph].

[13] TWiki: LHC SUSY Cross Section Working Group, https://twiki.cern.ch/twiki/bin/ view/LHCPhysics/SUSYCrossSections\#Cross_sections_for_various_subpr. Accessed: 13.08.2019.

[14] GitHub repository: fuenfundachtzig/xsec, https://github.com/fuenfundachtzig/xsec. Accessed: 13.08.2019.

[15] ATLAS Collaboration, Search for squarks and gluinos in final states with hadronically decaying $\tau$-leptons, jets, and missing transverse momentum using pp collisions at $\sqrt{s}=13 \mathrm{TeV}$ with the ATLAS detector, Phys. Rev. D 99 (2019) 012009, arXiv:1808.06358 [hep-ex] .

[16] ATLAS Collaboration, Search for top squarks decaying to tau sleptons in pp collisions at $\sqrt{s}=13$ TeV with the ATLAS detector, Phys. Rev. D 98 (2018) 032008, arXiv: 1803.10178 [hep-ex].

[17] C. G. Lester and D. J. Summers, Measuring masses of semi-invisibly decaying particles pair produced at hadron colliders, Phys. Lett. B 463 (1999) 99-103, arXiv: hep-ph/9906349 [hep-ph] .

[18] A. Barr, C. Lester, and P. Stephens, A variable for measuring masses at hadron colliders when missing energy is expected; $m_{T 2}$ : the truth behind the glamour, J. Phys. G 29 (2003) 2343-2363, arXiv:hep-ph/0304226.

[19] C. G. Lester and B. Nachman, Bisection-based asymmetric $M_{T 2}$ computation: a higher precision calculator than existing symmetric methods, JHEP 03 (2015) 100, arXiv: 1411.4312 [hep-ph].

[20] CMS Collaboration, Search for top squark pair production in a di-tau final state in proton-proton collisions at $\sqrt{s}=13 \mathrm{TeV}$, CMS-PAS-SUS-19-003, 2019, http: //cds.cern.ch/record/2676094.

[21] CMS Collaboration, Search for supersymmetry in events with a $\tau$ lepton pair and missing transverse momentum in proton-proton collisions at $\sqrt{s}=13 \mathrm{TeV}$, JHEP 11 (2018) 151, arXiv: 1807.02048 [hep-ex].

[22] ATLAS Collaboration, Search for the direct production of charginos and neutralinos in final states with tau leptons in $\sqrt{s}=13 \mathrm{TeV}$ pp collisions with the ATLAS detector, Eur. Phys. J. C 78 (2018) 154, arXiv:1708.07875 [hep-ex].

[23] The LEP SUSY Working Group and the ALEPH, DELPHI, L3 and OPAL experiments note LEPSUSYWG/04-01, http: / / lepsusy . web. cern. ch/lepsusy/Wel come.html.

[24] ALEPH Collaboration, Search for scalar leptons in $e^{+} e^{-}$collisions at centre-of-mass energies up to 209 GeV, Phys. Lett. B 526 (2002) 206. 
[25] ALEPH Collaboration, Absolute mass lower limit for the lightest neutralino of the MSSM from $e^{+} e^{-}$ data at $\sqrt{s}$ up to $209 \mathrm{GeV}$, Phys. Lett. B 583 (2004) 247.

[26] DELPHI Collaboration, Searches for supersymmetric particles in $e^{+} e^{-}$collisions up to $208 \mathrm{GeV}$ and interpretation of the results within the MSSM, Eur. Phys. J. C 31 (2003) 421.

[27] L3 Collaboration, Search for scalar leptons and scalar quarks at LEP, Phys. Lett. B 580 (2004) 37.

[28] OPAL Collaboration, Search for anomalous production of di-lepton events with missing transverse momentum in $e^{+} e^{-}$collisions at $\sqrt{s}=183-209 \mathrm{GeV}$, Eur. Phys. J. C 32 (2004) 453.

[29] ATLAS Collaboration, Search for direct production of charginos, neutralinos and sleptons in final states with two leptons and missing transverse momentum in pp collisions at $\sqrt{s}=8 \mathrm{TeV}$ with the ATLAS detector, JHEP 05 (2014) 071, arXiv: 1403.5294 [hep-ex] .

[30] ATLAS Collaboration, Search for the electroweak production of supersymmetric particles in $\sqrt{s}=8 T e V$ pp collisions with the ATLAS detector, Phys. Rev. D 93 (2016) 052002, arXiv:1509.07152 [hep-ex].

[31] ATLAS Collaboration, Search for the direct production of charginos, neutralinos and staus in final states with at least two hadronically decaying taus and missing transverse momentum in pp collisions at $\sqrt{s}=8 \mathrm{TeV}$ with the ATLAS detector, JHEP 10 (2014) 096, arXiv:1407.0350 [hep-ex] .

[32] ATLAS Collaboration, Search for heavy charged long-lived particles in the ATLAS detector in $31.6 \mathrm{fb}^{-1}$ of proton-proton collision data at $\sqrt{\mathrm{s}}=13 \mathrm{TeV}$, Phys. Rev. D 99 (2019) 092007, arXiv:1902.01636 [hep-ex].

[33] CMS Collaboration, Search for direct $\tau$ slepton pair production in proton-proton collisions at $\sqrt{s}=13 \mathrm{TeV}$, CMS-PAS-SUS-18-006, 2019, https : / / cds. cern. ch/record/2669190.

[34] ATLAS Collaboration, Search for direct stau production in events with two hadronic tau leptons in $\sqrt{s}=13 \mathrm{TeV}$ p p collisions with the ATLAS detector, ATLAS-CONF-2019-018, 2019, http: / / cdsweb. cern. ch/record/2676595.

[35] ATLAS Collaboration, Prospect for a search for direct stau production in events with at least two hadronic taus and missing transverse momentum at the High Luminosity LHC with the ATLAS Detector, ATL-PHYS-PUB-2016-021, 2016, https : / / cds . cern. ch/record/2220805.

[36] CMS Collaboration, Search for supersymmetry with direct stau production at the HL-LHC with the CMS Phase-2 detector, CMS-PAS-FTR-18-010, 2019, https: //cds.cern. ch/record/2647985.

[37] G. Apollinari, I. Béjar Alonso, O. Brüning, M. Lamont, and L. Rossi, High-Luminosity Large Hadron Collider (HL-LHC): Preliminary Design Report. CERN Yellow Reports: Monographs. 2015. http://cds.cern. ch/record/2116337.

[38] G. Apollinari, I. Béjar Alonso, O. Brüning, P. Fessia, M. Lamont, L. Rossi, and L. Tavian, High-Luminosity Large Hadron Collider (HL-LHC): Technical Design Report V. 0.1. CERN Yellow Reports: Monographs. 2017. https: / / cds. cern. ch/record/2284929. 